East Asian Math. J.

Vol. 30 (2014), No. 5, pp. 609-616

http://dx.doi.org/10.7858/eamj.2014.040

\title{
HYPERBOLIC EQUATIONS RELATED TO $p$-LAPLACIAN OPERATORS VIA TIME DISCRETIZATION METHODS
}

\author{
KiYeon Shin and Sujin Kang
}

\begin{abstract}
In this paper we consider hyperbolic equations related to the $p$-Laplacian with a nonsmooth kernel. By exploiting a suitable implicit time-discretization technique we obtain the existence of global strong solution.
\end{abstract}

\section{Introduction}

A variety of phenomena arising in elasticity theory, molecular dynamics, mechanics and quantum mechanics leads to nonlinear partial differential equations of second order in time. Thus, in this paper, we study a hyperbolic differential equation related to the $p$-Laplacian operator. More precisely, we are interested in the existence and uniqueness of the solution of problem

$$
\begin{array}{ll}
u_{t t}+\beta\left(u_{t}\right)-\Delta_{p} u \ni f(t) & \text { in } \Omega \times[0, T], \\
u=0 & \text { on } \partial \Omega \times[0, T], \\
u(\cdot, 0)=u^{0}, \quad u^{\prime}(\cdot, 0)=v^{0} & \text { in } \Omega,
\end{array}
$$

where $-\Delta_{p} u=-\operatorname{div}\left(|\nabla u|^{p-2} \nabla u\right), \beta$ is linear growth condition and $f \in L^{\infty}(\Omega)$. $T>0$ denotes some reference final time and $\Omega$ is a regular open bounded set of $\mathbb{R}^{d}(d \geq 1)$ with its boundary $\partial \Omega$.

The functional analytic formulation of (1) leads to the initial value problem $u^{\prime \prime}+A u^{\prime}+B u=f, u(0)=u^{0}, u^{\prime}(0)=v^{0}$. For the case that $A$ is maximal monotone and $B$ is linear, the existence and uniqueness of the solution has been established by Lions and Strauss [6] by using a Faedo-Galerkin method. Another proof has been given by Brézis [4] in the theory of nonlinear semigroups. In this paper, we prove the the existence and uniqueness of the solution in case that $A$ is linear and $B$ is nonlinear by time-discretization method. It should be remarked that such a result applies to various significant cases of hyperbolic partial differential equations in refer to [6], [7].

Received April 14, 2014; Accepted August 1, 2014.

2000 Mathematics Subject Classification. 2000 Mathematics Subject Classification: $45 \mathrm{~K} 05,35 \mathrm{~K} 55,35 \mathrm{~K} 45$.

Key words and phrases. Hyperbolic equation, p-Laplacian, Time discretization.

This work was supported by a 2-Year Research Grant of Pusan National University. 
The object of our work is not only to establish existence and uniqueness properties for the solution of (1), but also to introduce a discrete approximation of the problem, then prove convergence of the discrete solution to the continuous one. Thus we recall our assumptions and state main results in Section 2. In Section 3, we show the existence of discrete scheme. After showing some estimates on the approximations, the passage to the limit and the existence results are given in Section 4 .

\section{Assumptions and Main results}

We let $\|\cdot\|_{p},\|\cdot\|_{1, p}$ and $\|\cdot\|_{-1, p}$ denote the norm in $L^{p}(\Omega), W_{0}^{1, p}(\Omega)$ and $W^{-1, p}(\Omega)$ for $1<p<\infty$, respectively $([1])$. And $<\cdot, \cdot>$ denotes the duality between $W_{0}^{1, p}(\Omega)$ and $W^{-1, p}(\Omega)$ or denotes inner product of $L^{2}(\Omega)$. For $1<$ $p<\infty$, we define the conjugate $p^{\prime}$ of $p$ by $1 / p+1 / p^{\prime}=1$. In this paper, $C_{i}$ and $C$ will denote positive constants and $\lambda_{i}$ the imbedding constants of [1].

Now, we present our assumptions which are used throughout this paper. We suppose $d^{*} \leq p<\infty$ where $d^{*}=(2 d) /(d+2), u^{0} \in L^{\infty}(\Omega)$ with $u^{0}=0$ on $\partial \Omega, v^{0} \in L^{\infty}(\Omega)$ with $v^{0}=0$ on $\partial \Omega$ and the following:

(H) The function $\beta: \mathbb{R} \rightarrow \mathbb{R}$ is linear with $(\beta(u)) u \geq 0$ and $|\beta(u)| \leq C u$ where $C$ is a positive constant.

Definition 1. ([2]) Let $X$ be a reflexive Banach space and $A: X \rightarrow X^{\prime}$. We say that $A$ is monotone if $\langle A y-A z, y-z\rangle \geq 0$ for all $y, z \in X$, and $A$ is hemicontinuous if for each $y, z, w \in X$ the real-valued function $t \rightarrow$ $\langle A(y+t z), w\rangle$ is continuous.

Lemma 2.1. (Minty's Theorem [9]) Let $X$ be a reflexive Banach space. If $A: X \rightarrow X^{\prime}$ is monotone and hemicontinuous, then

$$
A y=f \quad \text { if and only if } \quad\langle f-A z, y-z\rangle \geq 0
$$

for all $z \in X$.

Lemma 2.2. ([3]) Let $\Omega$ be a bounded set in $\mathbb{R}^{d}$. Let $1<p<\infty$ be fixed and $A: W_{0}^{1, p}(\Omega) \rightarrow W^{1, p^{\prime}}(\Omega)$ a nonlinear operator defined by

$$
A(u)=-\operatorname{div} a(x, u, D u)
$$

where $a(x, s, \xi)$ is a Carathéodory function $a: \Omega \times \mathbb{R} \times \mathbb{R}^{d} \rightarrow \mathbb{R}^{d}$ such that

$$
\begin{aligned}
& |a(x, s, \xi)| \leq \beta\left[|s|^{p-1}+|\xi|^{p-1}+k(x)\right], \\
& {[a(x, s, \xi)-a(x, s, \eta)](\xi-\eta)>0, \quad \xi \neq \eta,} \\
& a(x, s, \xi) \xi \geq \alpha|\xi|^{p},
\end{aligned}
$$

where $k(x) \in L^{p^{\prime}}(\Omega), k \geq 0, \beta>0$ and $\alpha>0$.

Let $g(x, s, \xi)$ be a Carathéodory function such that

$$
\begin{aligned}
& g(x, s, \xi) s \geq 0, \\
& |g(x, s, \xi)| \leq b(|s|)\left(|\xi|^{p}+c(x)\right),
\end{aligned}
$$


where $b$ is a continuous and increasing function with (finite) values on $\mathbb{R}^{+}$and $c \in L^{1}(\Omega), c \geq 0$. Then, for $h \in W^{-1, p^{\prime}}(\Omega)$, the problem

$$
A u+g(x, u, \nabla u)=h,
$$

has at least one solution $u \in W_{0}^{1, p}(\Omega)$.

Lemma 2.3. ([8]) If $u \in W_{0}^{1, p}(\Omega)$ is a solution to the equation

$$
-\Delta_{p} u+F(x, u)=h,
$$

where $h \in W^{-1, r}, r>\frac{d}{p-1}$ and $F$ satisfies $\xi F(x, \xi) \geq 0$ in $\Omega \times \mathbb{R}$, then $u \in L^{\infty}(\Omega)$.

Now, we state our main results as follows :

Theorem 2.4. Under the assumptions $(\mathrm{H})$, there exists a unique solution $u$ of (1) such that $u \in L^{p}\left(0, T ; W_{0}^{1, p}(\Omega)\right) \cap L^{\infty}\left(0, T ; L^{\infty}(\Omega)\right)$.

\section{Existence of scheme}

For the problem (1), we consider the discrete scheme (DS) for $i=1,2, \ldots, N$,

$$
(\mathrm{DS}) \quad\left(\begin{array}{ll}
\frac{v_{i}-v_{i-1}}{\tau}+\beta\left(\frac{u_{i}-u_{i-1}}{\tau}\right)-\Delta_{p} u_{i}=f_{i} & \text { in } \Omega, \\
\frac{u_{i}-u_{i-1}}{\tau}=v_{i} & \text { in } \partial \Omega, \\
u_{i}=0, v_{i}=0 & \text { in } \Omega .
\end{array}\right.
$$

where $N \tau=T$ and $T$ is a fixed positive real. We shall show that (DS) has a solution $u_{i}$ for $i=1,2, \ldots, N$.

Theorem 3.1. Let $(\mathrm{H})$ hold. Then for $i=1,2, \ldots, N$, there exists a unique solution $u_{i} \in W_{0}^{1, p}(\Omega) \cap L^{\infty}(\Omega)$ of (DS) for sufficiently small $\tau$.

Proof. First of all, we rewrite (DS) as

$$
-\tau^{2} \Delta_{p} u_{i}+F\left(x, u_{i}\right)=\varphi_{i-1}
$$

where $F\left(x, u_{i}\right)=(1+\tau \beta)\left(u_{i}\right)$ and $\varphi_{i-1}=u_{i-1}-\tau v_{i-1}+\tau \beta\left(u_{i-1}\right)+\tau^{2} f_{i}$.

Now, we consider the equation

$$
-\tau^{2} \Delta_{p} u+F(x, u)=\varphi_{1}=u_{0}-\tau v_{0}+\tau \beta\left(u_{0}\right)+\tau^{2} f_{1},
$$

where $F(x, u)=(1+\tau \beta)(u)$ for fixed $\tau=T / N$. It is obvious that $a(x, u, D u):=$ $|\nabla u|^{p-2} \nabla u$ satisfies all the three conditions of $a$ in Lemma 2.3 (cf [3]). And, by assumption, Lemma 2.3 and Lemma 2.4, there exists a solution $u \in W_{0}^{1, p}(\Omega) \cap$ $L^{\infty}(\Omega)$. We put $u_{1}:=u$ and we obtain $v_{1}$ by scheme. Then we consider the equation $-\tau \Delta_{p} u+F(x, u)=\varphi_{2}=u_{1}-\tau v_{1}+\tau \beta\left(u_{1}\right)+\tau^{2} f_{2}$ where $F(x, u)=$ $(1+\tau \beta)(u)$. Continuing this process, we have a solution $u_{i}$ of (DS) for $i=$ $1,2, \ldots, N$ such that $u_{i} \in W_{0}^{1, p}(\Omega) \cap L^{\infty}(\Omega)(i=1,2, \ldots, N)$. 
Next, we show the uniqueness of $u_{i}(i=1,2, \ldots, N)$. Let $u_{i}$ and $u_{i}^{*}$ be two solutions of (DS) for $i=1,2, \ldots, N$. Using the result which we will establish below (see Theorem 3.2), we have

$$
\left\|u_{i}\right\|_{1, p}+\left\|u_{i}^{*}\right\|_{1, p} \leq M
$$

where $M$ is a suitable positive constant independent of $\tau$. And, from [5] we have

$$
\left\langle-\Delta_{p} u+\Delta_{p} v, u-v\right\rangle \geq C_{p}\|u-v\|_{1, p}^{p}
$$

for all $u, v \in W_{0}^{1, p}(\Omega)$.

Since $u_{i}$ and $u_{i}^{*}$ are solutions of (DS), we have

$$
-\tau^{2} \Delta_{p} u_{i}+\tau^{2} \Delta_{p} u_{i}^{*}+(I+\tau \beta)\left(u_{i}-u_{i}^{*}\right)=0 .
$$

Multiplying (4) by $u_{i}-u_{i}^{*}$ and integrating over $\Omega$, we get

$$
\left\langle-\tau^{2} \Delta_{p} u_{i}+\tau^{2} \Delta_{p} u_{i}^{*}, u_{i}-u_{i}^{*}\right\rangle+\int_{\Omega}\left((I+\tau \beta)\left(u_{i}-u_{i}^{*}\right)\right)\left(u_{i}-u_{i}^{*}\right) d x=0 .
$$

By (3) and $(\mathrm{H})$, the equation (5) becomes

$$
\left(\tau^{2} C_{p}-\tau C_{p}\right)\left\|u_{i}-u_{i}^{*}\right\|_{1, p}^{p} \leq-\frac{1}{2} \int_{\Omega}\left|u_{i}-u_{i}^{*}\right|^{2} d x .
$$

Thus, it implies that for sufficiently small $\tau$, i.e., $\tau<\frac{1}{C_{p}}, u_{i}=u_{i}^{*}$ holds.

Now, we consider the bounds of $\left\{u_{i}\right\}(i=1,2, \ldots, N)$, which is constructed in Theorem 3.1 as solutions of (DS).

Theorem 3.2. We assume $(\mathrm{H})$. Then there exist $C_{1}-C_{3}$, which are positive constants and independent of $\tau$, such that for all $i=1,2, \ldots, m$,

$$
\begin{aligned}
& \text { (a) }\left\|v_{i}\right\|_{\infty} \leq C_{1}, \\
& \text { (b) } \tau \sum_{i=1}^{m}\left\|u_{i}\right\|_{1, p}^{p} \leq C_{2}, \\
& \text { (c) }\left\|v_{m}\right\|_{2}^{2}+\sum_{i=1}^{m}\left\|v_{i}-v_{i-1}\right\|_{2}^{2} \leq C_{3},
\end{aligned}
$$

where $m=1,2, \ldots, N$.

Proof. (a) Multiplying (DS) by $\left|v_{i}\right|^{k} v_{i}$ and integrating over $\Omega$, we have by (H), $f \in L^{\infty}(\Omega)$ and Hölder's inequality,

$$
\int_{\Omega}\left|v_{i}\right|^{k} v_{i} v_{i} d x \leq\left\|v_{i}\right\|_{k+2}^{k+1}\left\|v_{i-1}\right\|_{k+2}+m(\Omega)^{1 /(k+2)} \tau C_{4}\left\|v_{i}\right\|_{k+2}^{k+1} .
$$

Then $\left\|v_{i}\right\|_{k+2} \leq m(\Omega)^{1 / k+2} \tau C_{4}+\left\|v_{i-1}\right\|_{k+2}$. By induction, we have $\left\|v_{i}\right\|_{k+2} \leq$ $m(\Omega)^{1 /(k+2)} C_{4} T+\left\|v_{0}\right\|_{k+2}$. Letting $k \rightarrow \infty,\left\|v_{i}\right\|_{\infty} \leq C\left(C_{4}, T, u_{0}\right)=: C_{1}$. 
(b) Let $z \in W_{0}^{1, p}(\Omega)$ be fixed. Multiplying the equation (DS) by $u_{i}-z$ and integrating over $\Omega$, we have

$$
\begin{aligned}
& \left\langle v_{i}-v_{i-1}, u_{i}\right\rangle+\tau\left\|u_{i}\right\|_{1, p}^{p} \\
& \quad=\left\langle v_{i}-v_{i-1}, z\right\rangle-\tau\left\langle\Delta_{p} u_{i}, z\right\rangle-\tau\left\langle\beta\left(v_{i}\right), u_{i}-z\right\rangle+\left\langle f_{i}, u_{i}-z\right\rangle .
\end{aligned}
$$

Now, we apply Young's inequality to (6) to get

$$
\begin{aligned}
& \left\langle v_{i}-v_{i-1}, u_{i}\right\rangle+\frac{\tau}{4}\left\|u_{i}\right\|_{1, p}^{p} \\
& \quad \leq\left\langle v_{i}-v_{i-1}, z\right\rangle+\tau\left\{\left(\frac{1}{4} \frac{p}{p-1}\right)^{-(p-1)} p^{-1}+C C_{1} \lambda+C_{f} \lambda\right\}\|z\|_{1, p}^{p} \\
& +\tau\left(\frac{1}{4} \frac{p}{\lambda_{1}}\right)^{-\frac{p}{p^{\prime}}}\left(p^{\prime}\right)^{-1} C_{f}^{p^{\prime}} m(\Omega)+\tau\left(C_{1} C\right)^{p^{\prime}} m(\Omega)+\left(\frac{p}{4 \lambda_{1}}\right)^{-\frac{p}{p^{\prime}}}\left(p^{\prime}\right)
\end{aligned}
$$

Now we define $\varphi(v)=\langle v, v\rangle / 2$ and $\varphi^{*}(v)=\sup _{z}\{\langle v, z\rangle-\varphi(z)\}$, then we have

$$
\begin{aligned}
& \varphi^{*}\left(v_{i}\right)-\varphi^{*}\left(v_{i-1}\right)+\frac{\tau}{4}\left\|u_{i}\right\|_{1, p}^{p} \\
& \quad \leq\left\langle v_{i}-v_{i-1}, z\right\rangle+\tau C\left(\|z\|_{1, p}, p, \lambda, \lambda_{1}, m(\Omega), C, C_{f}, C_{1}\right)+\tau^{2} \sum_{j=1}^{i} \tau\left\|u_{j}\right\|_{1, p}^{p}
\end{aligned}
$$

for $i=1,2, \ldots, m$ and for arbitrary $m=1,2, \ldots, N$. By summing (7) for $i=1,2, \ldots, m$,

$$
\begin{aligned}
& \varphi^{*}\left(v_{m}\right)-\left\langle v_{m}, z\right\rangle+\frac{\tau}{4} \sum_{i=1}^{m}\left\|u_{i}\right\|_{1, p}^{p} \\
& \quad \leq \varphi^{*}\left(v_{0}\right)-\left\langle v_{0}, z\right\rangle+C_{5}+\tau \sum_{i=1}^{m} \sum_{j=1}^{i} \tau\left\|u_{j}\right\|_{1, p}^{p},
\end{aligned}
$$

where $C_{5}:=C\left(\|z\|_{1, p}, p, \lambda, \lambda_{1}, m(\Omega), C, C_{f}, C_{1}\right)$ for $m=1,2, \ldots, N$. Applying the discrete Gronwall's lemma to (8),

$\varphi^{*}\left(v_{m}\right)-\left\langle v_{m}, z\right\rangle+\frac{\tau}{4} \sum_{i=1}^{m}\left\|u_{i}\right\|_{1, p}^{p} \leq C\left(v_{0},\|z\|_{1, p}, p, \lambda, \lambda_{1}, m(\Omega), C, C_{f}, C_{1}\right)$.

Hence $\tau \sum_{i=1}^{m}\left\|u_{i}\right\|_{1, p}^{p} \leq C_{3}$.

(c) Multiplying (DS) by $v_{i}$ and integrating over $\Omega$, we get by $(\mathrm{H})$ and using $a(a-b)=\frac{1}{2} a^{2}-\frac{1}{2} b^{2}+\frac{1}{2}(a-b)^{2}$,

$$
\left\|v_{i}\right\|_{2}^{2}-\left\|v_{i-1}\right\|_{2}^{2}+\left\|v_{i}-v_{i-1}\right\|_{2}^{2} \leq \tau\left(1+C_{f}\right) C_{1} .
$$

Summing the above inequality for $i=1,2, \ldots, m$, we have

$$
\left\|v_{m}\right\|_{2}^{2}+\sum_{i=1}^{m}\left\|v_{i}-v_{i-1}\right\|_{2}^{2} \leq T\left(1+C_{f}\right) C_{1}+\left\|v_{0}\right\|_{2}^{2},
$$


for $m=1,2, \ldots, N$. Thus, $\left\|v_{m}\right\|_{2}^{2}+\sum_{i=1}^{m}\left\|v_{i}-v_{i-1}\right\|_{2}^{2} \leq C_{3}$.

In the forthcoming discussion, the following notations will be used extensively. For vectors $u_{i}(i=0,1, \ldots, N)$ in Theorem 3.1, we define two functions $u_{\tau}$ and $\bar{u}_{\tau}$ on $[0, T]$ by

$$
\begin{array}{ll}
u_{\tau}(0):=u_{0}, & u_{\tau}(t):=u_{i}+\frac{u_{i}-u_{i-1}}{\tau}(t-i \tau), \\
\bar{u}_{\tau}(0):=u_{0}, & \bar{u}_{\tau}(t):=u_{i},
\end{array}
$$

for $t \in((i-1) \tau, i \tau](i=1,2, \ldots, N)$ and $\tau=T / N$. Similarly, we define

$$
\begin{array}{ll}
v_{\tau}(0):=v_{0}, & v_{\tau}(t):=v_{i}+\frac{v_{i}-v_{i-1}}{\tau}(t-i \tau), \\
\bar{v}_{\tau}(0):=v_{0}, & \bar{v}_{\tau}(t):=v_{i},
\end{array}
$$

for $i=0,1, \ldots, N$. Also, we let $\bar{f}_{\tau}(t):=f_{i}$ for $t \in((i-1) \tau, i \tau](i=1,2, \ldots, N)$.

Hence we can rewrite (DS) in a more compact form as

$$
v_{\tau}^{\prime}+\beta\left(u_{\tau}^{\prime}\right)-\Delta_{p} \bar{u}_{\tau}+\bar{f}_{\tau}=0, \quad \text { a.e. } \quad \text { in }[0, T] .
$$

\section{Estimates and Limits}

\subsection{Estimates}

First of all, by the consequence of Theorem 3.2, the followings are very easily proven.

$$
\begin{aligned}
& \bar{u}_{\tau} \text { is bounded in } L^{p}\left(0, T ; W_{0}^{1, p}(\Omega)\right) \cap L^{\infty}\left(0, T ; L^{\infty}(\Omega)\right), \\
& u_{\tau}^{\prime} \text { is bounded in } L^{p}\left(0, T ; W_{0}^{1, p}(\Omega)\right), \\
& \bar{v}_{\tau} \text { is bounded in } L^{\infty}\left(0, T ; L^{\infty}(\Omega)\right), \\
& v_{\tau}^{\prime} \text { is bounded in } L^{2}\left(0, T ; L^{\infty}(\Omega)\right) .
\end{aligned}
$$

Moreover, by (10) and boundedness of $p$-Laplacian operator,

$$
-\Delta_{p} \bar{u}_{\tau} \text { is bounded in } L^{p^{\prime}}\left(0, T ; W^{-1, p^{\prime}}(\Omega)\right) .
$$

And by (9), (11), (13) and $f \in L^{\infty}(\Omega)$,

$$
v_{\tau}^{\prime} \text { is bounded in } L^{p^{\prime}}\left(0, T ; W^{-1, p^{\prime}}(\Omega)\right) .
$$

We emphasis that all of the above boundedness are independent of $\tau$.

First of all, we have $u \in L^{p}\left(0, T ; W_{0}^{1, p}(\Omega)\right) \cap L^{\infty}\left(0, T ; L^{\infty}(\Omega)\right)$ which is a weak limit of $\bar{u}_{\tau}$ as $\tau \rightarrow 0$ by (10). i.e., $\bar{u}_{\tau}$ converges weakly to $u$ as $\tau \rightarrow 0$ in $L^{p}\left(0, T ; W_{0}^{1, p}(\Omega)\right) \cap L^{\infty}\left(0, T ; L^{\infty}(\Omega)\right)$. 


\subsection{Limits and Proof of Theorem 2.4}

As we mentioned in (10)-(15), we have $u, v$ and $f$ such that

$$
\begin{aligned}
& \bar{u}_{\tau} \rightarrow u \quad \text { weakly in } \quad L^{p}\left(0, T ; W_{0}^{1, p}(\Omega)\right) \cap L^{\infty}\left(0, T ; L^{\infty}(\Omega)\right), \\
& v_{\tau} \rightarrow v \quad \text { weakly star in } \quad W^{1, p^{\prime}}\left(0, T ; W^{-1, p^{\prime}}(\Omega)\right), \\
& v_{\tau} \rightarrow v \quad \text { weakly in } C\left(0, T ; L^{2}(\Omega)\right), \\
& \bar{v}_{\tau} \rightarrow v \quad \text { weakly star in } \quad L^{p^{\prime}}\left(0, T ; W^{-1, p^{\prime}}(\Omega)\right), \\
& \bar{v}_{\tau} \rightarrow v \quad \text { strongly in } \quad L^{2}\left(0, T ; L^{2}(\Omega)\right), \\
& \bar{f}_{\tau} \rightarrow f \quad \text { strongly in } \quad L^{p^{\prime}}\left(0, T ; W_{0}^{-1, p^{\prime}}(\Omega)\right) .
\end{aligned}
$$

We note that the above sequences with $\tau$ are for some not relabeled subsequence. Also we note that

$$
\begin{aligned}
\lim _{\tau \rightarrow 0} \int_{0}^{T}\left\langle\phi, \bar{v}_{\tau}-v_{\tau}\right\rangle d t \leq \lim _{\tau \rightarrow 0} \tau \sum_{i=1}^{N} \int_{(i-1) \tau}^{i \tau} \int_{\Omega} \phi(x, t)\left(\frac{v_{i}-v_{i-1}}{\tau}\right) d x d t \\
\quad=\lim _{\tau \rightarrow 0} \tau \sum_{i=1}^{N} \int_{(i-1) \tau}^{i \tau} \int_{\Omega} \phi(x, t) v_{\tau}^{\prime}(t) d x d t \\
\quad=\lim _{\tau \rightarrow 0} \tau \int_{0}^{T}\left\langle\phi, v_{\tau}^{\prime}\right\rangle d t=0,
\end{aligned}
$$

for all $\phi \in L^{p}\left(0, T ; W_{0}^{1, p}(\Omega)\right)$ by $(15)$. Hence, $v_{\tau}$ and $\bar{v}_{\tau}$ have the same limit $v$ in (17) and (18).

Therefore,

$$
-\Delta_{p} \bar{u}_{\tau} \rightarrow-u_{t t}-\beta\left(u_{t}\right)-f \text { weakly in } L^{p^{\prime}}\left(0, T ; W_{0}^{-1, p^{\prime}}(\Omega)\right) .
$$

And

$$
\begin{aligned}
& \limsup _{\tau \rightarrow 0} \int_{0}^{T}\left\langle-\Delta_{p} \bar{u}_{\tau}, \bar{u}_{\tau}\right\rangle d t \leq \limsup _{\tau \rightarrow 0} \int_{0}^{T} \int_{\Omega}-v_{\tau}^{\prime} \bar{u}_{\tau} d x d t \\
& +\limsup _{\tau \rightarrow 0} \int_{0}^{T} \int_{\Omega}-\beta\left(u_{\tau}^{\prime}\right) \bar{u}_{\tau} d x d t+\limsup _{\tau \rightarrow 0} \int_{0}^{T} \int_{\Omega}-\bar{f}_{\tau} \bar{u}_{\tau} d x d t .
\end{aligned}
$$

Therefore, by Lemma 2.1 (Minty's Theorem), (14) and (16)-(20), there exist a unique solution $u$ of $(1)$ such that $u \in L^{p}\left(0, T ; W_{0}^{1, p}(\Omega)\right) \cap L^{\infty}\left(0, T ; L^{\infty}(\Omega)\right)$ if $p \geq 2$.

\section{References}

[1] Adams, R., Sobolev Spaces, Academic Press, New York, 1975.

[2] Barbu, V., Nonlinear semigroups and differential equations in Banach spaces, Noordhoff Internat. Publ., Leyden, 1976.

[3] Bensoussan, A., Boccardo, L., Murat, F., On a nonlinear P.D.E. having natural growth terms and unbounded solutions, Ann. Inst. H. Poincare 5 (1988), no.4, 347-364.

[4] Brézis, H., Semi-groups non linéaires et applications, Sympos. Math. 7, Academic Press, London, 1971. 
[5] Le, V., Schmit, K., Global Bifurcation in Variational Inequalities, Springer-Verlag, New York, 1997.

[6] Lions, J.L., Quelques méthodes de résolution des problémes aux limites non liéaires, Dunod Gauthier-Villars, Paris, 1969.

[7] Lions, J.L., Strauss, W.A., Some non-linear evolution equations, Bull. Soc. Math. France, 9 (1965), 43-96.

[8] J.M. Rakotoson, On some degenerate and nondegenerate Quasilinear Elliptic systems with nonhomogeneous Dirichlet boundary condition, Nonlinear Analysis, T.M.A., 13 (1989), no.2, 165-183.

[9] R.E. Showalter, Monotone operators in Banach space and nonlinear partial differential equations, Mathematical Surveys and Monographs 49, AMS, 1996.

KIYEON SHIN

Department of Mathematics, Pusan National University, Pusan, 609-735, Korea

E-mail address: kyshin@pusan.ac.kr

SUJIN KANG

Department of Nanomaterials Engineering, Pusan National University,

Pusan, 609-735, Korea

E-mail address: sjnisj@pusan.ac.kr 